\title{
Soft-Bit Assisted Iterative AMR-WB Source-Decoding and Turbo-Detection of Channel-Coded Differential Space-Time Spreading Using Sphere Packing Modulation
}

\author{
N. S. Othman, M. El-Hajjar, O. Alamri and L. Hanzo* \\ School of ECS, University of Southampton, SO17 1BJ, UK. \\ Tel: +44-23-8059 3125, Fax: +44-23-8059 4508 \\ Email: $\{1 \mathrm{~h}\} @$ ecs.soton.ac.uk, http://www-mobile.ecs.soton.ac.uk
}

\begin{abstract}
Jointly optimised iterative source- and channel-decoding is used for enhancing the error resilience of the Adaptive Multi Rate WideBand (AMR-WB) speech codec. The resultant AMR-WB coded speech signal is protected by Recursive Systematic Convolutional (RSC) codes and transmitted using a Sphere Packing (SP) aided Differential Space-Time Spreading (DSTS) assisted transceiver. The performance of the proposed scheme is evaluated when communicating over correlated non-dispersive Rayleigh fading channels. The proposed system exhibits an $E_{b} / N_{0}$ gain of about $1 \mathrm{~dB}$ in comparison to the benchmark scheme carrying out joint channel decoding and DSTS aided SP-demodulation, but separate AMR-WB decoding, when using $I=4$ external iterations.
\end{abstract}

\section{MOTIVATION AND BACKGROUND}

The classic Shannonian source and channel coding separation theorem [1] has limited applicability in the context of finite-complexity, finite-delay lossy speech [2] and video [3] codecs, where the different encoded bits exhibit different error sensitivity. These arguments are particularly valid, when the limited-complexity, limiteddelay source encoders fail to remove all the redundancy from the correlated speech or video source signal. Fortunately, this residual redundancy may be beneficially exploited for error protection by intelligently exchanging soft information amongst the various receiver components. More explicitly, it was explicitly demonstrated in [4] that the video performance of a twin-class protected MPEG4 video transceiver substantially benefitted from a multi-stage turbo detection process, which exchanged soft-information across three Soft-In-SoftOut (SISO) blocks.

These powerful turbo principles may be further enchanced by exploiting the innovative concept of soft speech bits, which was developed by Vary and his team $[5,6]$, culminating in the formulation of iterative source and channel decoding (ISCD) [7]. More explicitly, in ISCD the source- and channel-decoder iteratively exchange $a$ priori and extrinsic information for the sake of improving the overall system performance. As a further development, in [8] the employment of the turbo principle [9] for iterative soft demapping in multilevel modulation [10] schemes combined with channel coding demonstrated an enhanced bit error rate performance. Thus, ISCD combined with iterative soft demapping in the context of multilevel modulation and amalgamated with a number of other sophisticated wireless transceiver components may beneficially exploited. In the resultant

The financial support of the Universiti Tenaga Nasional Malaysia, of Vodafone under the auspices of the Dorothy Hodgkin Postgraduate Award, of the Ministry of Higher Education of Saudi Arabia and that of the EPSRC, UK as well as that of the European Union in the framework of the Pheonix and Newcom projects is gratefully acknowledged. multi-stage scheme extrinsic information is exchanged amongst three receiver components, namely the demodulator, the channel decoder and the soft-input source decoder in the spirit of [11].

Explicitly, we proposed and investigate a jointly optimised ISCD scheme invoking the Adaptive Multirate-Wideband (AMR-WB) speech codec [12], which is protected by a Recursive Systematic Convolutional (RSC) code. The resultant bitstream is transmitted using Differential Space-Time Spreading (DSTS) and Sphere Packing (SP) modulation [13] over a correlated, non-dispersive Rayleigh fading channel. An efficient iterative turbo-detection scheme is utilised for exchanging extrinsic and a priori information between the constituent codes. In an effort to mitigate the effects of the hostile Rayleigh fading channel, DSTS employing two transmit and one receive antennas was invoked for sake of providing a spatial diversity gain. This powerful wireless transceiver is advocated, since it was demonstrated that the employment of SP modulation combined with the orthogonal transmit diversity designs of [14], outperformed the conventional orthogonal design based Space-Time Block Coding (STBC) schemes of $[15,16]$.

The paper is structured as follows. In Section 2, the AMR-WB speech codec is described briefly and the residual redundancy inherent in its parameters is quantified. In Section 3, the overall system model is described, while our simulation results are presented in Section 4. Our conclusions are offered in Section 5.

\section{RESIDUAL REDUNDANCY IN THE AMR-WB SPEECH CODEC}

The AMR-WB speech codec is capable of supporting nine different bit rates [17], each of which may be activated in conjunction with different-rate channel codecs and different-throughput adaptive modem modes [18]. Similar near-instantaneously adaptive speech and video systems were designed in $[2,3]$. In our prototype system investigated here the AMR-WB codec operated at $15.85 \mathrm{kbit} / \mathrm{s}$ extracts a set of speech parameters per $20 \mathrm{~ms}$ frame for representing the speech signal. Each AMR-WB frame consists of 317 bits per $20 \mathrm{~ms}$ of speech frame. Similar to any other code excited linear prediction (CELP) based codec [2], it performs short-term linear prediction (LP), longterm prediction (LTP) and generates the excitation codebook parameters [12]. The resultant bit-allocation scheme is summarized in Table 1. The LP coefficients produced for the $20 \mathrm{~ms}$ speech frame are converted to the so-called immittance spectrum pair (ISP) representation [19] and they are vector-quantised using split-multistage vector quantisation (S-MSVQ) producing 7 ISP parameters. The LTP analysis [2] is performed for each new $5 \mathrm{~ms}$ subframe, which produces the LTP lag and gain parameters. The LTP lag is encoded using 9 bits in the first and third subframes, and differentially encoded with respect to the first and third subframes, using 6 bits in the second and fourth subframes, respectively. The LTP and fixed code- 


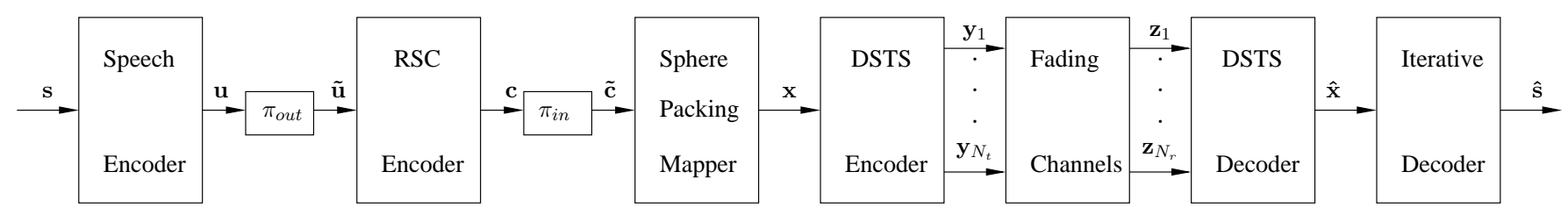

Figure 1: Block diagram of the DSTS-SP-RSC-AMRWB scheme. The notations $\mathbf{s}, \hat{\mathbf{s}}, \mathbf{u}, \mathbf{c}, \mathbf{x}, \mathbf{y}_{j}, \mathbf{z}_{k}, \pi_{\text {out }}$ and $\pi_{\text {in }}$ represent the frame of the speech source samples, the estimate of the speech source samples, the bit-representation of the encoded speech parameters, the encoded bits of the RSC encoder, the SP-coded symbols, the DSTS coded symbols of transmitter $j$, the received symbols at receiver $k$, the outer bit interleaver, and the inner bit interleaver, respectively. Furthermore, $N_{t}$ and $N_{r}$ denote the number of transmitters and receivers, respectively. The iterative decoder seen at the right is detailed in Figure 2.

\begin{tabular}{|c|c|c|c|c|c|}
\hline Parameter & $\begin{array}{c}1 \mathrm{st} \\
\text { subfr }\end{array}$ & $\begin{array}{l}\text { 2nd } \\
\text { subfr }\end{array}$ & $\begin{array}{l}\text { 3rd } \\
\text { subfr }\end{array}$ & $\begin{array}{l}\text { 4th } \\
\text { subfr }\end{array}$ & $\begin{array}{c}\text { Total } \\
\text { per frame }\end{array}$ \\
\hline VAD-flag & & & & & 1 \\
\hline ISPs & & & & & 46 \\
\hline LTP Lag & 9 & 6 & 9 & 6 & 30 \\
\hline LTP-filt.-flag & 1 & 1 & 1 & 1 & 4 \\
\hline Fixed Index & 52 & 52 & 52 & 52 & 208 \\
\hline CB Gains & 7 & 7 & 7 & 7 & 28 \\
\hline Total & & & & & 317 \\
\hline
\end{tabular}

7, 5 and 5 bits, respectively. Additionally, we assume that the ISPs corresponding to the same ISP index $i=1, \ldots, 7$ in the other AMR-WB encoded frames are independent and each of the ISPs within a frame exhibits first-order Markov chain, so,

$$
\begin{aligned}
& P\left(U_{n}=u_{n} \mid U_{1}=u_{1}, \ldots, U_{n-1}=u_{n-1}\right) \\
& \quad=P\left(U_{n}=u_{n} \mid U_{n-1}=u_{n-1}\right)
\end{aligned}
$$

Following the derivation in [21], $H(\mathcal{U})$ can be expressed as:

$$
\begin{aligned}
H(\mathcal{U})= & \lim _{n \rightarrow \infty} H\left(U_{n} \mid U_{1}, U_{2}, \ldots, U_{n-1}\right) \\
= & \lim _{n \rightarrow \infty} H\left(U_{n} \mid U_{n-1}\right) \\
= & H\left(U_{n} \mid U_{n-1}\right) \\
= & \mathcal{E}_{p\left(u_{n}, u_{n-1}\right)} \\
& \times \log _{2}\left[P\left(U_{n}=u_{n} \mid U_{n-1}=u_{n-1}\right)\right]
\end{aligned}
$$

book gains are jointly vector quantized using 7 bits per subframe,
whilst the fixed excitation codebook parameters of each $5 \mathrm{~ms}$ subframe are encoded using 13 bits. [17]. A large training sequence of 2,133,035 $20 \mathrm{~ms}$ frames was applied to the AMR-WB encoder in order to estimate the residual redundancy of each individual quantised AMR-WB parameter. In general, the residual redundancy inherent in the encoded bit-stream representing the speech signal at the output of the speech encoder reflects the residual redundancy manifesting itself in terms of the residual correlation (or memory). Another sign of substantial residual redundancy is the non-uniform probability of occurance for the various encoded speech parameters. Let us assume that each of the encoded codec parameters can be sufficiently accurately modelled by a stationary stochastic process and each of the encoded parameters is modelled by a zero-order-or a first-order Markov chain, when quantifying the residual redundancy manifesting itself in terms of the nonuniform relative frequency of the legitimate encoded speech parameters. Let us now consider the estimation of the residual redundancy inherent in the AMR-WB encoded parameters which is quantified by estimating the entropy $H(\mathcal{U})$ of the encoded parameters, where $\mathcal{U}$ represents the random process modelling the AMR-WB encoded parameters. The entropy $H(\mathcal{U})$ of the encoded parameters, represents the minimum number of bits required for describing the parameter. Thus, if each parameter is encoded by $\mathcal{B}$ bits/symbol, the total residual redundancy inherent in the parameter is given by $\mathcal{R}_{T}=\mathcal{B}-H(\mathcal{U})$. The residual redundancy reflects the residual correlation (or memory), $\mathcal{R}_{M}$ and nonuniformity, $\mathcal{R}_{D}$. Specifically, we can write $\mathcal{R}_{T}=\mathcal{R}_{D}+\mathcal{R}_{M}$ where for variable $U_{1}$, the redundancy due to the nonuniformity $\mathcal{R}_{D}=\mathcal{B}-H\left(U_{1}\right)$, whilst, $\mathcal{R}_{M}=H\left(U_{1}\right)-H(\mathcal{U})$ denotes the redundancy due to the memory [20]. Let us provide an example for quantifying the entropy $H(\mathcal{U})$ of the ISP parameters. Assuming the ISP parameters can be approximated by a stationary stochastic process, let the random process, $U_{i}: i=1,2, \ldots, 7$, represent the $i^{t h}$ vector quantized ISP in each AMR-WB frame, which are encoded using 8, 8, 6, 7,

Thus, the total residual redundancy, $\mathcal{R}_{T}$ of $U_{2}$ is given by:

$$
\mathcal{R}_{T}=8-H(\mathcal{U})
$$

since, $\mathcal{R}_{T}=\mathcal{R}_{D}+\mathcal{R}_{M}$, thus, giving:

$$
\begin{aligned}
\mathcal{R}_{D} & =8-H\left(U_{2}\right) \\
\mathcal{R}_{M} & =H\left(U_{2}\right)-H\left(U_{2} \mid U_{1}\right)
\end{aligned}
$$

where $H\left(U_{2}\right)$ is the entropy of parameter $U_{2}$, given by:

$$
H\left(U_{2}\right)=-\sum_{u_{1}} P\left(U_{2}=u_{2}\right) \log _{2}\left[P\left(U_{2}=u_{2}\right)\right]
$$

The total redundancy of each AMR-WB codec parameter modelled with the aid of a first-order as a Markov Chain is shown in Table 2, although, the total redundancy of the fixed codebook index was modelled by a zero-order Markov chain, as shown in Table 3. When communicating the second and fifth columns of Table 2 as well as the second and fourth columns of Table 3, it becomes clear that the AMRWB codec is highly efficient in removing the redundancy. Hence, we may conclude from Table 2 and 3, that the residual correlation of all parameters within a given frame is insignificant and hence its exploitation is unlikely to be justifiable, considering the significant complexity imposed in terms of their trellis decoding invoked for exploiting our related first-order a priori knowledge, since the attainable performance would only be marginally increased. Hence, only our zero-order a priori knowledge is exploited in the decoding process. It will be shown in Section 4 that the exploitation of this residual redundancy at the decoder has the potential of providing substantial performance gains when compared to the receiver dispensing with this knowledge. 


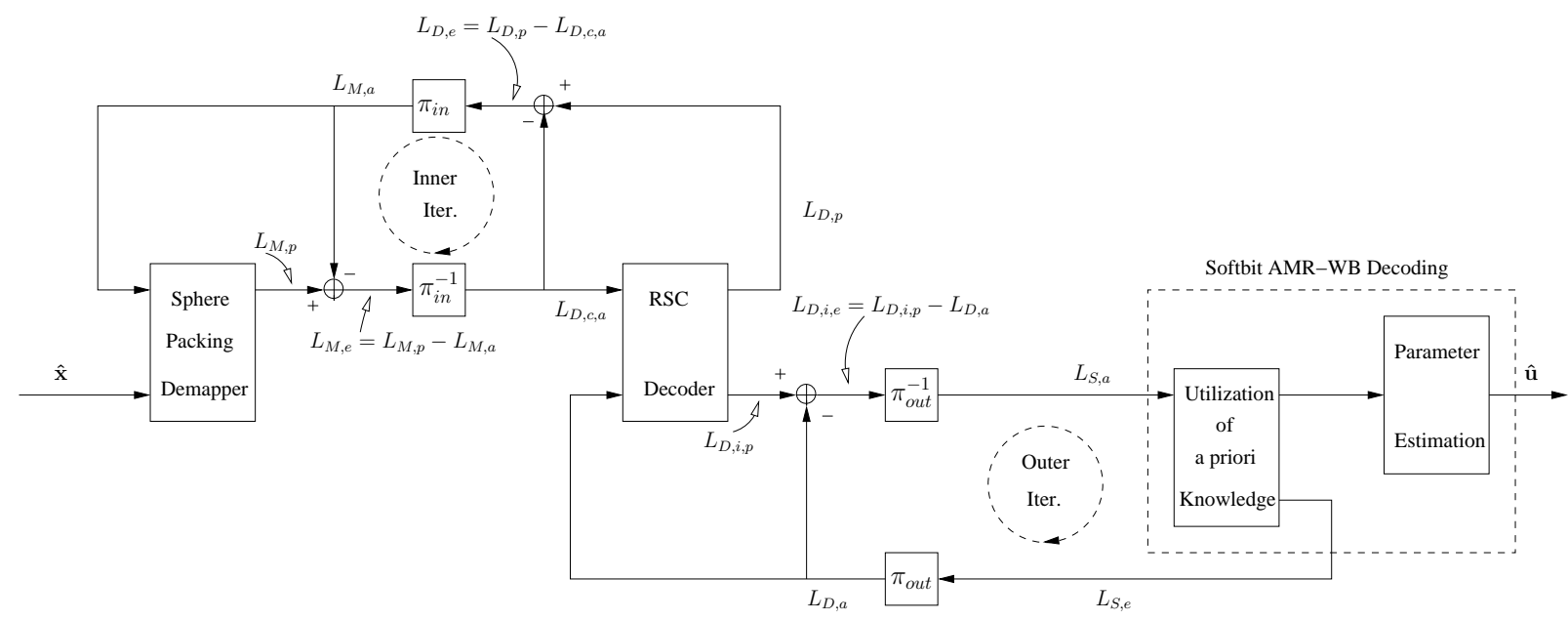

Figure 2: Block diagram of the jointly optimised DSTS-SP-RSC-AMRWB turbo detection scheme seen at the right of Figure 1. The notations $\pi_{i n}$ and $\pi_{\text {in }}^{-1}$ represent the inner interleaver and deinterleaver, $\pi_{\text {out }}$ and $\pi_{\text {out }}^{-1}$ denote the outer interleaver and deinterleaver.

\begin{tabular}{|cc|c|c|c|c|}
\hline \multicolumn{2}{|c|}{ Parameter } & Encoded Bits & $\mathcal{R}_{D}$ & $\mathcal{R}_{M}$ & $\mathcal{R}_{T}$ \\
\hline ISP No. & 1 & 8 & 0.57 & 0.00 & 0.57 \\
& 2 & 8 & 0.45 & 0.96 & 1.41 \\
& 3 & 6 & 0.10 & 0.10 & 0.20 \\
& 4 & 7 & 0.06 & 0.06 & 0.12 \\
& 5 & 7 & 0.14 & 0.05 & 0.19 \\
& 6 & 5 & 0.03 & 0.03 & 0.06 \\
\hline LTP Lag & subfr 1 & 5 & 0.07 & 0.04 & 0.11 \\
& subfr 2 & 6 & 0.23 & 0.00 & 0.23 \\
& subfr 3 & 9 & 0.40 & 0.21 & 0.61 \\
& subfr 4 & 6 & 0.39 & 0.14 & 0.34 \\
CB Gain & subfr 1 & 7 & 0.10 & 0.00 & 0.60 \\
& subfr 2 & 7 & 0.10 & 1.10 & 1.20 \\
& subfr 3 & 7 & 0.10 & 1.12 & 1.22 \\
& subfr 4 & 7 & 0.12 & 1.12 & 1.24 \\
\hline
\end{tabular}

Table 2: Residual Redundancy in the AMR-WB codec's Parameters Modelled by a First-Order Markov Chain

\begin{tabular}{|cl|c|c|c|}
\hline Parameter & & Encoded Bits & $\mathcal{R}_{D}$ & $\mathcal{R}_{T}$ \\
\hline Fixed Ind. subfr1 & 1 & 13 & 0.33 & 0.33 \\
& 2 & 13 & 0.31 & 0.31 \\
& 3 & 13 & 0.31 & 0.31 \\
& 4 & 13 & 0.31 & 0.31 \\
\hline
\end{tabular}

Table 3: Residual Redundancy in the Fixed Codebook Index Modelled by a Zero-Order Markov Chain

\section{SYSTEM OVERVIEW}

Figure 1 shows the schematic of the proposed scheme. The iterative decoder structure of the DSTS-SP-RSC-AMRWB scheme is illustrated in Figure 2, where the extrinsic information is exchanged amongst all three constituent decoders. The inner iterative loop corresponds to the iterative soft SP demapping and RSC channel decoding [13], while the outer iterative loop corresponds to the extrinsic/a priori information exchange between the AMR-WB speech decoder and the RSC decoder.

\subsection{Transmitter}

The AMR-WB speech encoder produces a frame of speech codec parameters, namely $\left\{\mathbf{v}_{1, \tau}, \mathbf{v}_{2, \tau}, \ldots, \mathbf{v}_{\kappa, \tau}, \ldots, \mathbf{v}_{36, \tau}\right\}$, where $\mathbf{v}_{\kappa, \tau}$ denotes an encoded parameter, with $\kappa=1, \ldots, K_{\kappa}$ denoting the position of each parameter in the encoded speech frame, whilst, $\tau$ denotes the time index referring to the current encoded frame index. Then, $\mathbf{v}_{\kappa, \tau}$ is quantised and mapped to the bit sequence, $\mathbf{u}_{\kappa, \tau}=\left[u(1)_{\kappa, \tau} u(2)_{\kappa, \tau}\right.$ $\left.\ldots u(M)_{\kappa, \tau}\right]$, where $M$ is the total number of bits assigned to the $\kappa$ th parameter. Then, the outer interleaver, $\pi_{\text {out }}$ permutes the bits of the sequence $\mathbf{u}$, yielding $\tilde{\mathbf{u}}$ of Figure 1 .

The interleaved RSC encoded source bit sequence $\tilde{\mathbf{u}}$ of Figure 1 is generated by a rate $R=\frac{1}{2}$ code having a constraint length of $K=7$ and octally represented generator polynomials of $\left(G_{r}, G\right)=$ $(217,110)$. The DSTS-SP modulator of Figure 1 first maps $B$ number of channel-coded bits $\tilde{\mathbf{c}}=\left[\begin{array}{lllll}\tilde{c}_{0} & \tilde{c}_{1} & \ldots & \tilde{c}_{B-1}\end{array}\right] \in 0,1$ to a sphere packing symbol $x \in X$, so that we have $x=\operatorname{map}_{s p}(\tilde{\mathbf{c}})$, where the function of $\operatorname{map}_{s p}(\tilde{\mathbf{c}})$ represents the mapping of $B$ bits to a SP symbol, $x$. Furthermore, we have $B=\log _{2} L=\log _{2} 16=4$, where $L$ represents the set of legitimate SP constellation points, as outlined in [14]. Subsequently, we have a set of SP symbols that can be transmitted using DSTS and two transmit antennas as detailed in [14]. Space-Time Spreading (STS) was first proposed by Marzetta et. al [22] for the sake of providing space-time-coding-style spatial transmit diversity gain for CDMA systems, as detailed in Section 8.2 of [23]. However, the channel impulse response (CIR) of all transmit-receive antenna links has to be estimated in coherently detected STS-CDMA systems which is a challenging task. Hence, in [13] the philosophy of differentially detected DSTS was developed, which dispenses with the need of estimating the CIR.

In this study, we considered transmissions over a correlated narrowband Rayleigh fading channel, associated with a normalised Doppler frequency of $f_{D}=0.01$.

\subsection{Receiver}

Inner Iterations: the complex-valued received symbols, $\mathbf{z}$ are de- 
mapped to their Log-likelihood Ratio (LLR) [9] representation for each of the $B$ RSC-encoded bits per DSTS-SP symbol. As seen in Figure 2, the a priori LLR values, $L_{M, a}$ provided by the RSC decoder are subtracted from the a posteriori LLR values, $L_{M, p}$ at the output of the SP-demapper for the sake of generating the extrinsic LLR values $L_{M, e}$ and then the LLRs $L_{M, e}$ are deinterleaved by a soft-bit deinterleaver. Next, the deinterleaved soft-bits $L_{D, c, a}$ of Figure 2 are passed to the RSC decoder in order to compute the a posteriori LLR values $L_{D, p}$ provided by the Max-log MAP algorithm [24] not only for the systematic bits, but for all the RSC-encoded bits. The extrinsic information $L_{D, e}$ seen in Figure 2 is generated by subtracting the a priori information, $L_{D, c, a}$ from the a posteriori information $L_{D, p}$ according to $L_{D, p}-L_{D, c, a}$, which is then fed back to the SP demapper as the a priori information $L_{M, a}$ after appropriately reordering them using the inner soft-value interleaver. The SP-demapper of Figure 2 exploits the a priori information $L_{M, a}$ for the sake of providing improved a posteriori LLR values, $L_{M, p}$ which are then passed to the RSC decoder and in task, back to the SP-demapper for further iterations.

Outer Iterations: As seen in Figure 2, the extrinsic LLR values $L_{D, i, e}$ of the original uncoded systematic information bits are generated by subtracting the a priori LLR values, $L_{D, a}$ of the RSC decoder from the LLR values $L_{D, i, p}$ of the original uncoded systematic information bits. Then, the LLRs $L_{D, i, e}$ are deinterleaved by the outer soft-bit deinterleaver. The resultant soft-bits $L_{S, a}$ are passed to the AMR-WB decoder that was further developed for handling soft input bits in order to compute the extrinsic LLR values, $L_{S, e}$ with the aid of soft-bit source decoding (SBSD). These extrinsic LLR values are then fed back to the RSC decoder after appropriately reordering them in the order required by the RSC decoder for the sake of completing an outer iteration. We define this as having $I=1$ iteration, namely, one external iteration. The residual redundancy quantified in Section 2 is exploited as a priori information in computing the extrinsic LLR values, and estimating the parameters.

The details of the algorithm used for computing the extrinsic LLR values, $L_{S, e}$ can be found in [7].

\section{PERFORMANCE RESULTS}

In this section we evaluate the attainable performance of the proposed scheme using both the Bit Error Ratio (BER) and the Segmental Signal to Noise Ratio (SegSNR) [2] evaluated at the speech decoder's output as a function of the channel SNR.

All simulation parameters are listed in Table 4. In our simulations each inner iteration was followed by an outer iteration.

Figure 3 depicts the BER versus Signal to Noise Ratio (SNR) per bit, namely $E_{b} / N_{0}$ performance of the non-coherent DSTS-SPRSC-AMRWB scheme and that of its corresponding DSTS-SP-RSC benchmarker scheme, when communicating over uncorrelated Rayleigh fading channels. It can be seen from Figure 3 that the DSTS-SPRSC-AMRWB scheme outperforms the DSTS-SP-RSC benchmarker scheme by about $1 \mathrm{~dB}$ at $\mathrm{BER}=1.5 \times 10^{-4}$ after $I=4$ iterations where, we define a complete decoding iteration cycle, as having an inner iteration followed by a single outer-iteration, as mentioned in Section 3, which is referred to as having $I=1$ iteration. The AMRWB-decoded scheme has a low BER at its speech decoded output than its benchmarker dispensing with speech decoding, because the extrinsic information exchange between the AMR-WB decoder and the RSC decoder has the potential of improving the BER.

Let us now study the speech SegSNR performance of the pro-

\begin{tabular}{|l|c|}
\hline System Parameters & DSTS-SP-RSC-AMR-WB \\
\hline \hline Source Coding & AMR-WB \\
\hline Bit Rates (kbit/s) & 15.85 \\
Speech Frame Length $(\mathrm{ms})$ & 20 \\
Sampling Rate $(\mathrm{kHz})$ & 16 \\
\hline Channel Coding & $R=1 / 2$ \\
\hline Code Rate & $K=7$ \\
Code Memory & $(217,110)_{8}$ \\
Code Generator $\left(G_{r}, G\right)$ & Recursive Sys. Conv. Code \\
\hline Modulation Scheme & 2 \\
\hline MIMO Scheme & 1 \\
\hline Number of Transmitters, $N_{t}$ & Walsh Code \\
Number of Receivers, $N_{r}$ & 8 \\
Spreading Code & 4 \\
Spreading Factor & Sphere Packing $(\mathrm{L}=16)$ \\
Number of users & Correlated Rayleigh Fading \\
\hline Channel & 0.01 \\
\hline Normalised Doppler frequency & 1 \\
\hline System Throughput $($ bit/symbol) & \\
\hline
\end{tabular}

Table 4: System Parameters

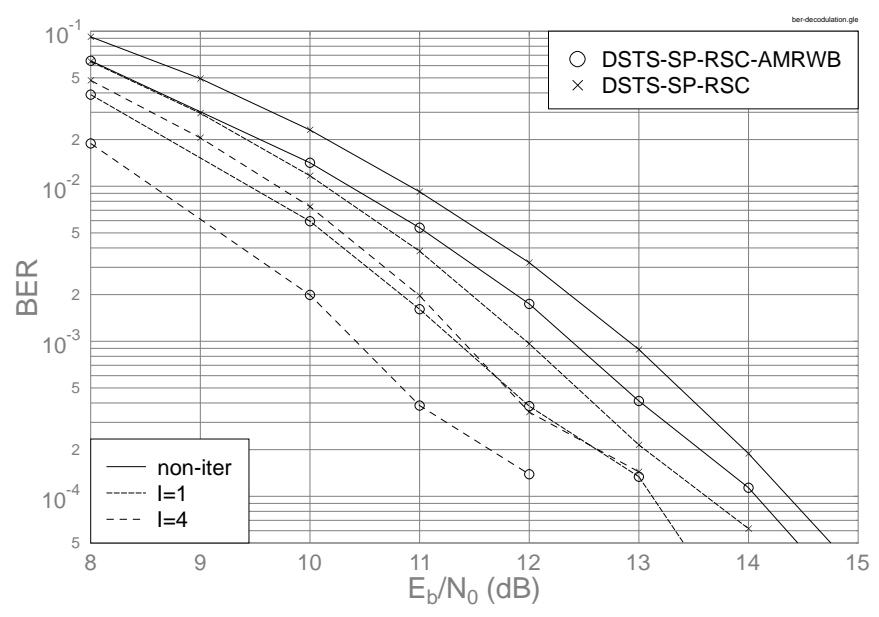

Figure 3: BER versus $E_{b} / N_{0}$ performance of the jointly optimised DSTS-SP-RSC-AMRWB scheme of Figure 1 and Table 4, when communicating over correlated non-dispersive Rayleigh fading channels.

posed scheme in Figure 4. In this context the residual redundancy inherent in the encoded source is exploited twice, firstly during computing the extrinsic information and secondly during the parameter Markov-model-based estimation [25]. It can be seen from Figure 4 that the exploitation of residual redundancy inherent in the encoded source during the decoding process benefitting from Markov-modelbased parameter estimation performs approximately $0.5 \mathrm{~dB}$ better in terms of the required channel $E_{b} / N_{0}$ value, than its corresponding hard speech decoding based counterpart, when allowing a SegSNR degradation of $1 \mathrm{~dB}$ in comparison to the maximum attainable SegSNR maintained over perfectly error-free channels. Additionally, iteratively exchanging the soft-information amongst the three receiver components of the amalgamated DSTS-SP-RSC-AMRWB scheme has resulted in a further $E_{b} / N_{0}$ gain of about $2.5 \mathrm{~dB}$ after $I=4$ iterations, when tolerating a SegSNR degradation of $1 \mathrm{~dB}$. 


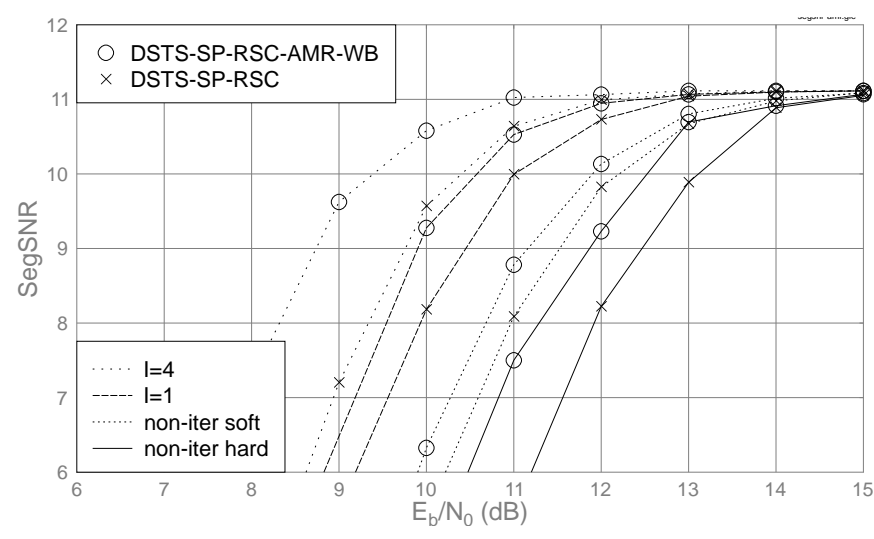

Figure 4: Average SegSNR versus $E_{b} / N_{0}$ performance of the jointly optimised DSTS-SP-RSC-AMRWB scheme of Figure 1 and Table 4 in comparison to the DSTS-SP-RSC benchmarker scheme, when communicating over correlated non-dispersive Rayleigh fading channels.

\section{CONCLUSIONS}

In this contribution the three-stage turbo detection DSTS-SP-RSCAMRWB scheme of Figure 1, 2 and Table 4 was proposed for transmission over a correlated non-dispersive Rayleigh fading channel. The employment of the soft-output AMR-WB speech codec, which exploits the residual redundancy inherent in the encoded bitstream demonstrates a significant improvement in terms of the average SegSNR versus channel $E_{b} / N_{0}$ performance compared to its corresponding hard decoding based benchmarker. The performance of the threecomponent turbo receiver is about $1 \mathrm{~dB}$ better in terms of the $E_{b} / N_{0}$ in comparison to the benchmarker scheme carrying out joint iterative channel decoding and DSTS aided SP-demodulation, but using separate AMR-WB decoding. The employment of the DSTS scheme that requires no channel state information, whilst providing a substantial diversity gain by invoking two transmit antennas.

\section{REFERENCES}

[1] C. E. Shannon, "A Mathematical Theory of Communication," The Bell System Technical Journal, vol. 27, pp. 379-423,623-656, July, October 1948

[2] L. Hanzo, F.C.A. Somerville, and J.P. Woodard, Voice Compression and Communications: Principles and Applications for Fixed and Wireless Channels. Chichester, UK: John Wiley-IEEE Press, 2001.

[3] L. Hanzo, P.J. Cherriman and J. Street, Wireless Video Communications: Second to Third Generation Systems and Beyond. NJ, USA : IEEE Press., 2001.

[4] S. X. Ng, J. Y. Chung and L. Hanzo, "Turbo-Detected Unequal Protection MPEG-4 Wireless Video Telephony Using Multi-Level Coding, Trellis Coded Modulation and Space-Time Trellis Coding," in IEE Communications Proceeding, vol. 152, pp. 1116-1124, 9 December 2005.

[5] T. Fingscheidt and P. Vary, "Speech Decoding with Error Concealment using Residual Source Redundancy," IEEE Workshop on Speech Coding for Telecommunication, pp. 91-92, 7-10 Sept 1997.

[6] T. Fingscheidt and P. Vary, "Softbit Speech Decoding: A New Approach to Error Concealment," IEEE Transactions on Speech and Audio Processing, vol. 9, pp. 240-251, March 2001.

[7] M. Adrat, P. Vary, and J.Spittka, "Iterative Source-Channel Decode Using Extrinsic Information from Softbit-Source Decoding," IEEE International Conference on Acoustics, Speech and Signal Processing, pp. 2653-2656, 7-11 May 2001.

[8] S. ten Brink, J. Speidel and R.-H. Yan, "Iterative Demapping and Decoding for Multilevel Modulation," IEEE Global Telecommunications Conference, vol. 1, pp. 579-584, November 1998.
[9] L. Hanzo, T. H. Liew and B. L. Yeap, Turbo Coding, Turbo Equalisation and Space Time Coding for Transmission over Wireless channels. New York, USA: John Wiley IEEE Press, 2002.

[10] L. Hanzo, Ng S. X, T.Keller and W. Webb, Quadrature Amplitude Modulation: From Basics to Adaptive Trellis-Coded, Turbo-Equalised and Space-Time Coded OFDM, CDMA and MC-CDMA Systems. New York, USA : John Wiley IEEE Press, 2004.

[11] T. Clevorn, J. Brauers, M. Adrat and P. Vary, "Turbo DeCodulation: Iterative Combined Demodulation and Source-Channel Decoding," IEEE Communications Letters, vol. 9, pp. 820-822, September 2005.

[12] B. Bessette, R. Salami, R. Lefebvre, M. Jelinek, J. Rotola-Pukkila, J. Vainio, H. Mikkola and K. Jarvinen, "The Adaptive Multirate Wideband Speech Codec (AMR-WB)," IEEE Transactions on Speech and Audio Processing, vol. 10, pp. 620-636, November 2002.

[13] M. El-Hajjar, O. Alamri, and L. Hanzo, "Differential Space-Time Spreading Using Iteratively Detected Sphere Packing Modulation and Two Transmit Antennas," in IEEE Wireless Communications and Networking Conference, vol. 3, pp. 1664-1668, April 2006.

[14] W.Su, Z.Safar and K.J.R. Liu, "Space-Time Signal Design for TimeCorrelated Rayleigh Fading Channels," IEEE International Conference on Communications, vol. 5, pp. 3175-3179, May 2003.

[15] S. Alamouti, "A Simple Transmit Diversity Technique for Wireless Communications," IEEE Journal on Selected Areas in Communications, vol. 16, pp. 1451-1458, October 1998.

[16] V. Tarokh, H. Jafarkhani, and A.R. Calderbank, "Space-Time Block Codes From Orthogonal Designs," IEEE Transactions on Information Theory, vol. 45, pp. 1456-1467, July 1999.

[17] 3GPP TS 26.190 V5, "AMR Wideband Speech Codec: Transcoding Functions," December 2001

[18] L. Hanzo, C. H. Wong and M. S. Yee, Adaptive Wireless Transceivers: Turbo-Coded, Turbo-Equalized and Space-Time Coded TDMA, CDMA, and OFDM Systems. New York, USA : John Wiley and Sons, 2002.

[19] Y. Bistritz and S. Pellerm, "Immitance Spectral Pairs (ISP) for Speech Encoding," IEEE International Conference on Acoustics, Speech, and Signal Processing (ICASSP), vol. 2, pp. 9-12, 27-30 April 1993.

[20] F. Alajaji, N. Phamdo, N. Farvardin, and T. E. Fuja, "Detection of Binary Markov Sources Over Channels with Additive Markov Noise," IEEE Transactions on Information Theory, vol. 42, pp. 230-239, January 1996.

[21] F. Alajaji, N. Phamdo, and T. E. Fuja, "Channel Codes that Exploit the Residual Redundancy in CELP-Encoded Speech," IEEE Transactions on Speech and Audio Processing, vol. 4, pp. 325-336, September 1996.

[22] B. Hochwald, T.L. Marzetta and C.B. Papadias, "A Transmitter Diversity Scheme for Wideband CDMA Systems Based on Space-Time Spreading," IEEE Journal on Selected Areas in Communications, vol. 19, pp. 48-60, September 2001.

[23] L. Hanzo, L-L. Yang, E-L. Kuan and K. Yen, Single and Multi-Carrier DS-CDMA: Multi-User Detection, Space-Time Spreading, Synchronisation, Networking and Standards. New York, USA : John Wiley and Sons, 2003.

[24] P. Robertson, E. Villebrun and P. Hoeher, "A Comparison of Optimal and Sub-Optimal MAP Decoding Algorithms Operating in the Log Domain," IEEE International Conference on Communications, vol. 2, pp. 10091013, 18-22 June 1995.

[25] T. Hindelang, T. Fingscheidt, N. Seshadri and R.V. Cox, "Combined source/channel (de-)coding: can a priori information be used twice?," IEEE International Conference on Communications, pp. 1208-1212, 1822 June 2000. 